\title{
Role of substrate on interaction of water molecules with graphene oxide and reduced graphene oxide
}

Roman Strzelczyk ${ }^{1,2}$, Cristina E. Giusca ${ }^{1 *}$, Francesco Perrozzi ${ }^{3}$, Giulia Fioravanti ${ }^{3}$, Luca Ottaviano $^{3}$, Olga Kazakova ${ }^{1}$

\author{
${ }^{1}$ National Physical Laboratory, Hampton Road, Teddington TW11 0LW, United Kingdom \\ ${ }^{2}$ Institute of Molecular Physics, Polish Academy of Sciences, Mariana Smoluchowskiego 17, \\ 60-179 Poznań, Poland \\ ${ }^{3}$ Dipartimento di Scienze Fisiche e Chimiche, Universita` dell'Aquila \& CNR-SPIN (UOS \\ L’Aquila), L’Aquila 67100, Italy
}

\begin{abstract}
We study local electronic properties of graphene oxide (GO) and reduced graphene oxide (RGO) on metallic $(\mathrm{Pt})$ and insulating $\left(\mathrm{Si}_{3} \mathrm{~N}_{4}\right)$ substrates in controlled humidity environment. We demonstrate that the supporting substrate plays a crucial role in interaction of these materials with water, with Pt making both GO and RGO insensitive to humidity variations and change in environment. On the other hand, in the case of $\mathrm{Si}_{3} \mathrm{~N}_{4}$ substrate a significant difference between GO and RGO with respect to humidity variations is demonstrated, indicating complete water coverage at $\sim 60 \%$ R.H for RGO and $\sim 30 \%$ R.H. for GO. Irrespective of the substrate, both GO and RGO demonstrate relative independence of their electronic properties on the material thickness, with similar trends observed for 1, 2 and 3 layers when subject to humidity variations. This indicates a relatively minor role of material thickness in GO-based humidity sensors.
\end{abstract}

\section{Introduction}

Graphene oxide (GO) is a disordered form of graphene containing functional oxygen groups, such as hydroxyl and epoxy, covalently attached to the basal $\mathrm{sp}^{2}$ plane of graphene, as well as carboxylic groups at the edges $[1,2,3,4,5]$. Presence of these groups turns the material into an electrical insulator. Reduction of the oxygen-containing groups by a relatively simple

\footnotetext{
*Corresponding author. E-mail: cristina.giusca@npl.co.uk
} 
thermal or chemical treatment results in reduced GO (RGO) [5]. This procedure allows to dramatically change the electrical properties of the material, converting it from an insulator to a semi-metal $[1,4,5]$.

Extensive research interest in GO and RGO owns to relatively easy and cheap production of the material, which is highly attractive for practical applications. For example, superpermeability of GO membranes to water molecules [6] opens a vast field for applications in water remediation and filtration [7]. In particular, GO functionalised by magnetite nanoparticles allows for magnetic-field assisted filtration of polluted water from dangerous substances containing heavy metal cations, see e.g. [8,9].

The large number of the reactive functional groups at the surface makes GO inherently attractive for environmental and biomedical sensing applications where such groups act as active anchoring sites for molecules [19, 20, 21, 22, 23]. On the other hand, the dielectric nature of GO is an obvious disadvantage for a sensor when used as a part of the electrical circuit [5]. As a compromise between graphene and GO in terms of chemical reactivity and electrical properties, RGO provides both the presence of reactive sites necessary for chemical reactivity $[10,11,12]$ as well as relatively high electrical conductivity. Additional advantages of GO/RGO for gas sensing include the material's inherent flexibility, transparency and suitability for large-scale manufacturing. In particular, the specific response of $\mathrm{GO}$ to $\mathrm{NO}_{2}$ was studied in various conditions and the sensitivity threshold of $\sim 20-50 \mathrm{ppb}$ was demonstrated [see, e.g. Refs. $5,13,14]$. The sensing ability was attributed to the presence of oxygen functional groups. Additionally, exceptionally high sensitivity to $\mathrm{NH}_{3}(\sim 1 \mathrm{ppb})$, accompanied by the large resistance change and fast sensor response, and combined with a selective response to many other chemical compounds [15] was reported in GO reduced by pyrrole.

Considering even more practical applications, bendable and washable electronic textile gas sensors made of yarn coated by RGO were recently demonstrated [16]. Such sensors demonstrate excellent chemical durability and mechanical stability combined with a high response to $\mathrm{NO}_{2}$ and selectivity to acetone, ethanol, ethylene, and $\mathrm{CO}_{2}$. Other advantages of etextiles include pliability, a light weight and low cost [17], which makes them desirable materials for high-performance flexible gas sensors [18]. GO was also successfully used in biosensing applications, e.g. protein [19], DNA [20] and enzymatic [21] detection, for regulation of neuronal signalling without affecting cell viability [22], as a biometric live-cell sensor [23] and many others.

A generally accepted electrical mechanism for gas sensing is consistent with the p-type conductivity, where a positively charged vacancy is created in the carbon lattice due to a 
negative charge transfer (a fraction of electron charge) from GO functional groups to the molecule. Additionally, unperturbed patches of graphene lattice can contribute through the intrinsic quantum capacitance of graphene.

In the case of water vapour, the dipolar nature of water molecules also adds to the overall spectrum of electrical effects $[5,24,25]$. The interaction of GO with water is particularly important due to the observed superpermeability of GO membranes [6] to water molecules as well as restricting conditions, which the presence of water imposes on gas sensing. In a number of cases, poorer sensitivity to gases is observed in humid conditions compared to dry gases, implying that water molecules form dipoles on the GO surface. The sensing mechanism in this case is based on the fact that reaction of water molecules with oxygen-containing functional groups releases protons, leading to an increase of conductivity $[12,26]$. Ultrafast GO-based humidity sensors were demonstrated, exhibiting such features as transparency, flexibility and easy upscaling, which makes them extremely attractive for applications [27, 28]. In particular, fast, touch-free sensors responding to modulation of moisture in a user's breath were demonstrated by Nokia for recognition applications [29].

Recently functional scanning probe microscopy was successfully used for studies of local electrical properties of various graphenes and 2D materials [30, 31, 32, 33, 34, 35], e.g. GO/RGO [36, 37, 38] These methods allow to study variations of properties on the nanometer scale, i.e. addressing differences in number of layers, non-homogeneous carrier density, screening and edge effects, local defect structure, etc. Knowledge of local electronic properties (e.g. obtained by scanning Kelvin probe microscopy (SKPM)) provides crucial information about the device performance, e.g. intrinsic resistivity, contact resistance, etc. [39]. Calibration of surface potential measurements and accurate determination of local work function were demonstrated $[32,35]$. In GO, correlation of work function and oxygen content [40] as well as their evolution under UV-radiation related to degradation of oxygen groups [41] were studied by SKPM.

In some cases, authors paid appropriate attention to hydrophilicity of GO and to the fact that water molecules form dipoles on the GO surface, thus affecting the correct determination of the local electrical properties (i.e. surface potential or dielectrical constant) [37,42]. Nevertheless, scanning experiments performed in a well-controlled environment and on welldefined samples are rare. In many previous studies combining local electrical measurements with a specific gas environment or level of humidity [43, 44] crucial factors such as film thickness, level of non-specific contamination and pre-conditioning of films helping to get rid of physisorbed molecules (e.g. by vacuum treatment and/or thermal annealing) were not 
considered, potentially leading to erroneous conclusions and generalization. Additionally, the effects of underlying substrates and related charge transfer were rarely taken into account. Furthermore, previous measurements of GO work function gave a very broad spread of the results, partly because work function is poorly defined in an insulator, but mainly due to unoptimised experimental conditions, i.e. lack of proper grounding of the sample, leading to charging of GO flakes.

The purpose of the current study is to investigate the effect of variable humidity $(0-70 \%$ $\mathrm{RH})$ on the local electronic properties (i.e. surface potential variation) of one-, two- and trilayer GO and RGO in a controllable manner, by means of SKPM. Additionally, we explore the role of supporting substrates (insulating vs metallic) in the charge transfer, as well as the cumulative effect of the substrate and environmental doping on the properties of GO/RGO. Understanding the role of water and taking into consideration GO thickness, will significantly facilitate development of various environmental and biological sensors.

\section{Experimental}

\subsection{GO Synthesis and reduction}

GO sheets were prepared via a modified Hummers method [45] and deposited by spin coating on $30 \mu \mathrm{m}$ wide platinum mesa structures, with $30 \mu \mathrm{m}$ lateral spacing in between electrodes, prefabricated on top of $\mathrm{Si}_{3} \mathrm{~N}_{4}$ substrates. Reduction of $\mathrm{GO}$ sheets was achieved by thermal annealing of the samples in ultra-high vacuum at $300{ }^{\circ} \mathrm{C}$. Typical lateral dimensions of GO and RGO sheets obtained via this method range from 40 to $100 \mu \mathrm{m}$, thus ensuring that a large number of individual flakes were placed both on $\mathrm{Si}_{3} \mathrm{~N}_{4}$ and Pt sections of the substrate, as confirmed by SEM, allowing to study their properties on both metallic and insulating substrates. Metallic mesa structure also allows for efficient grounding of the GO and RGO flakes, which is crucial, in particular, for conducting SPM measurements in vacuum.

\subsection{Scanning Electron Microscopy}

SEM images were obtained using the InLens detector of a Zeiss FESEM Supra system operated at $2 \mathrm{kV}$.

\subsection{Raman spectroscopy}

Raman intensity maps were obtained using a Horiba Jobin-Yvon HR800 system and a 532-nm laser excitation wavelength with $1 \mathrm{~mW}$ incident power. Data were taken with a spectral resolution of $(3.1 \pm 0.4) \mathrm{cm}^{-1}$ and lateral resolution of $(0.4 \pm 0.1) \mu \mathrm{m}$. 


\section{$2.4 S K P M$}

SKPM experiments were conducted in an environmental chamber using an NT-MDT Ntegra Aura SPM system and highly doped Si probes (PFQNE-AL, Bruker). Single-pass frequency-modulated SKPM (FM-SKPM) technique was used in all measurements. FMSKPM operates by detecting the force gradient $(d F / d z)$, which results in changes to the resonance frequency of the cantilever. In this technique, an AC voltage with a lower frequency $\left(f_{\text {mod }} \approx 3 \mathrm{kHz}\right)$ than the resonant frequency of the cantilever $\left(f_{o} \approx 300 \mathrm{kHz}\right)$ was applied to the probe, inducing a frequency shift of $f_{0} \pm f_{\text {mod }}$. The PID feedback loop monitors the side modes, $f_{0} \pm f_{\text {mod }}$, and compensates them by applying an offset DC voltage. By measuring the DC voltage at each pixel (equivalent to the contact potential difference $\left(\mathrm{U}_{\mathrm{CPD}}\right)$ ), a surface potential map is constructed. The same scanning probe (PFQNE-AL) was used for the entire sequence of the measurements presented in the paper.

\subsection{Environmental conditions}

FM-SKPM measurements in variable environmental conditions were performed in a controlled SPM chamber by monitoring the surface potential changes in the following sequence: (i) ambient (temperature and humidity of the laboratory ambient air: $\mathrm{T}=21^{\circ} \mathrm{C}$ and $40 \% \mathrm{RH}$, respectively); (ii) vacuum ( $\mathrm{P} \approx 1 \times 10^{-5} \mathrm{mbar}$ ); (iii) vacuum annealed state (with the sample cooled down to room temperature following thermal annealing at $150{ }^{\circ} \mathrm{C}$ for 1 hour); (iv) dry $\mathrm{N}_{2}$ atmosphere (research grade $99.9995 \%$ purity); (v) water vapour balanced with $\mathrm{N}_{2}$ with relative humidity in the range $10-70 \%$. For humidity exposure, RH was increased in a stepwise manner from $10 \%$ to $70 \%$ and the surface potential measurements were carried out immediately after reaching the target humidity level $\approx \approx 5 \mathrm{~min}$ at low $\mathrm{RH}$ and up to $\approx 30 \mathrm{~min}$ for $70 \% \mathrm{RH})$.

\section{Results and discussion}

The morphology and size distribution of as-prepared and reduced GO samples are first studied using SEM (Figure 1), which confirms that a large number of both GO and RGO sheets rest both on the $\mathrm{Si}_{3} \mathrm{~N}_{4}$ substrate and the Pt electrodes. Individual GO and RGO flakes in varying environmental conditions have been further studied using AFM/SKPM as described in the next 
sections. Raman spectroscopy was used to assess the degree of reduction of GO flakes and results are presented in the Supplementary Information (S.I.) section.
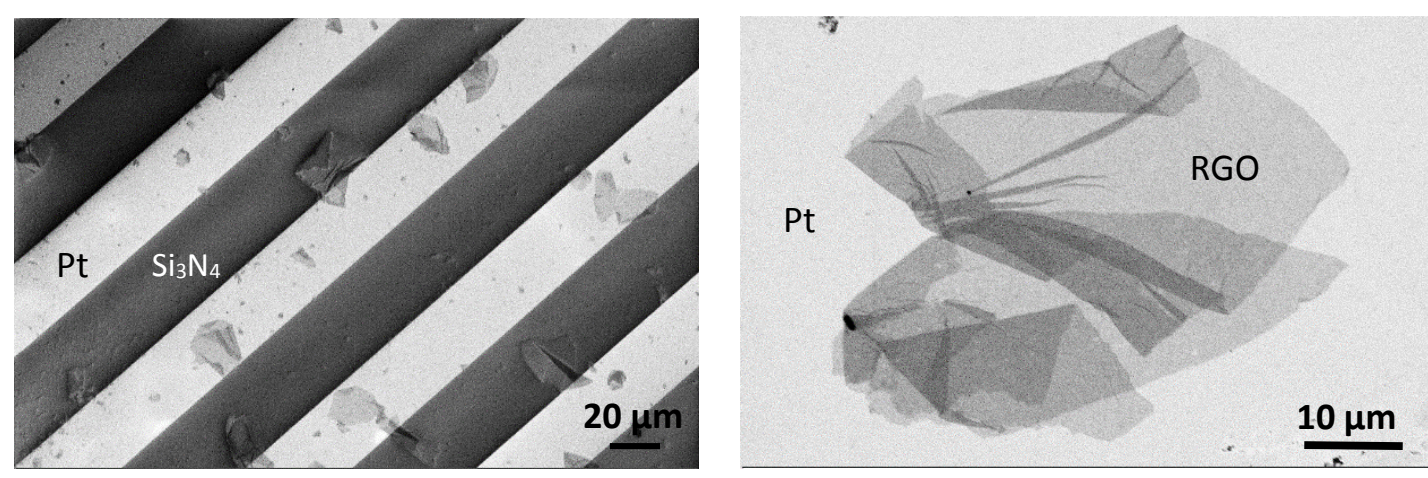

Figure 1: a) Representative SEM image of GO flakes on $\mathrm{Si}_{3} \mathrm{~N}_{4}$ substrate with $\mathrm{Pt}$ electrodes. Pt electrodes show bright contrast in the image; b) Individual RGO flake on Pt substrate.

\subsection{Reduced graphene oxide - humidity variations}

Figure 2a shows a topographic AFM image of RGO flakes, overlaying the border step (denoted by a dashed line) between silicon nitride and platinum areas of the substrate. The area of interest is chosen such that domains of different thickness (i.e. 1-3 layer (L) RGO) are positioned both on $\mathrm{Si}_{3} \mathrm{~N}_{4}$ and $\mathrm{Pt}$ areas, as indicated in the figure. Figures $2 \mathrm{~b}-\mathrm{f}$ show representative surface potential maps of the same area obtained in different environmental conditions, i.e. from ambient to vacuum, to the vacuum annealed state and then from low (10\% R.H.) to high (70\% R.H.) relative humidity. The humidity level was gradually increased in successive $10 \% \mathrm{RH}$ steps. All measurements are taken at room temperature. 

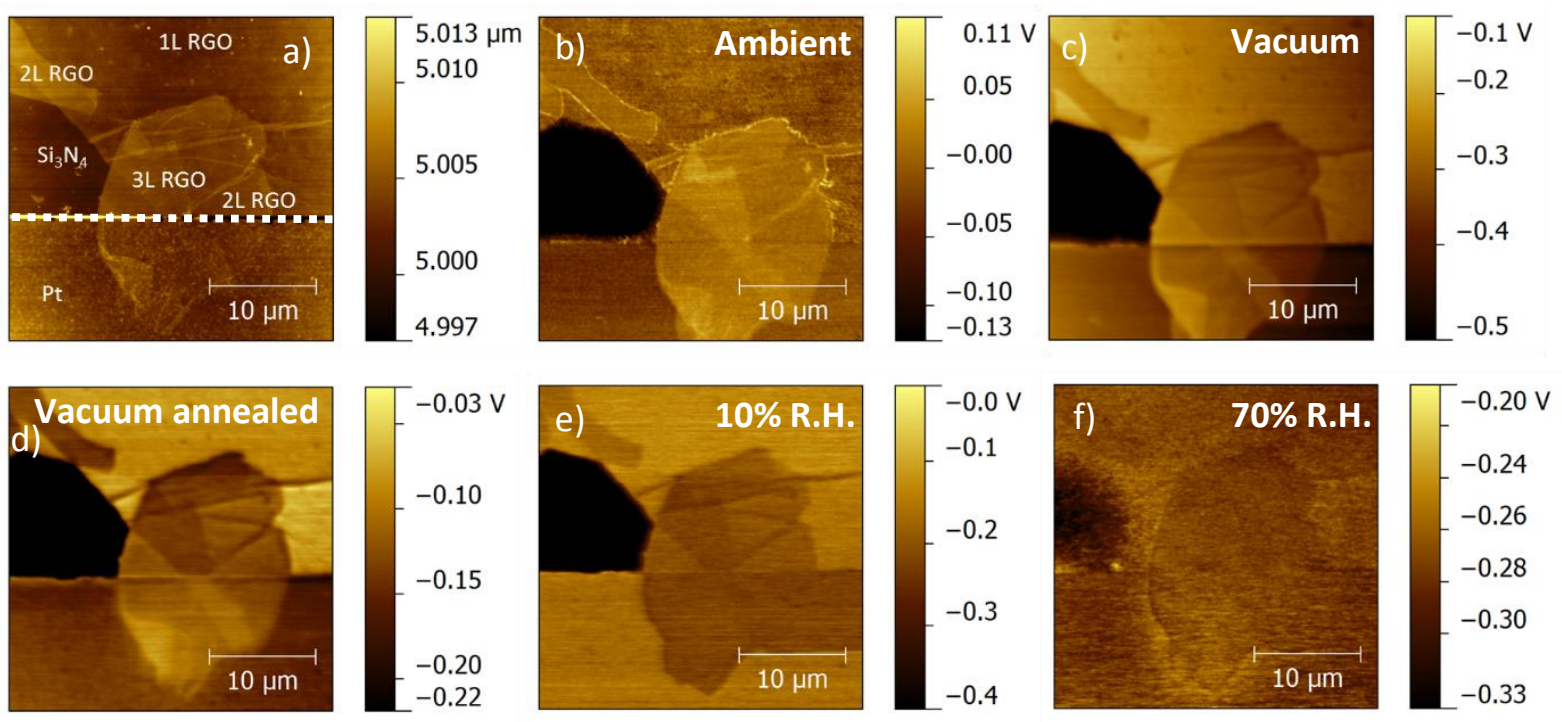

Figure 2: a) Topography and b-f) surface potential maps of RGO at different humidity levels: b) ambient; c) vacuum at RT; d) after thermal annealing in vacuum; e) $10 \%$ R.H. and f) $70 \%$ R.H. Underlying substrate and number of RGO layers are shown in a). Dashed line in a) indicates the border between $\mathrm{Si}_{3} \mathrm{~N}_{4}$ and $\mathrm{Pt}$ areas of the supporting substrate.

Values of the relative surface potential (i.e. the surface potential difference between individual domains and either $\mathrm{Si}_{3} \mathrm{~N}_{4}$ or Pt substrates) extracted from the images are summarised in Figures $3 \mathrm{a}$ and $\mathrm{b}$ with reference to $\mathrm{Si}_{3} \mathrm{~N}_{4}$ and Pt substrates, respectively.

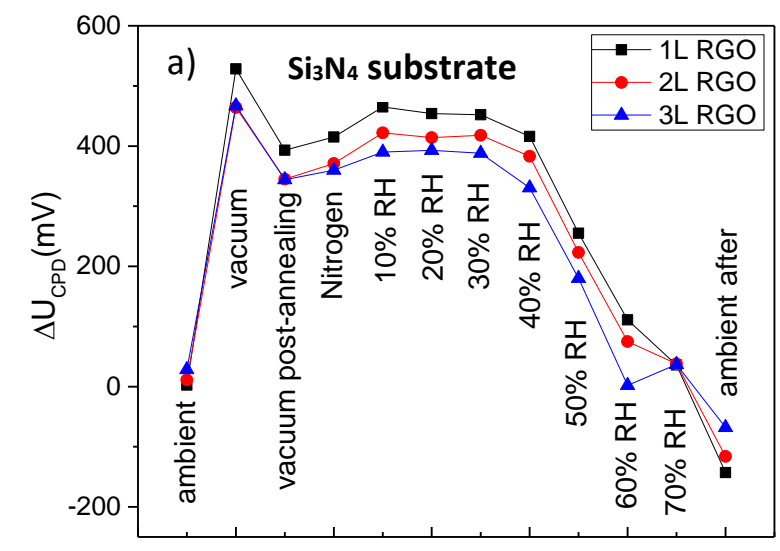

Measurement steps

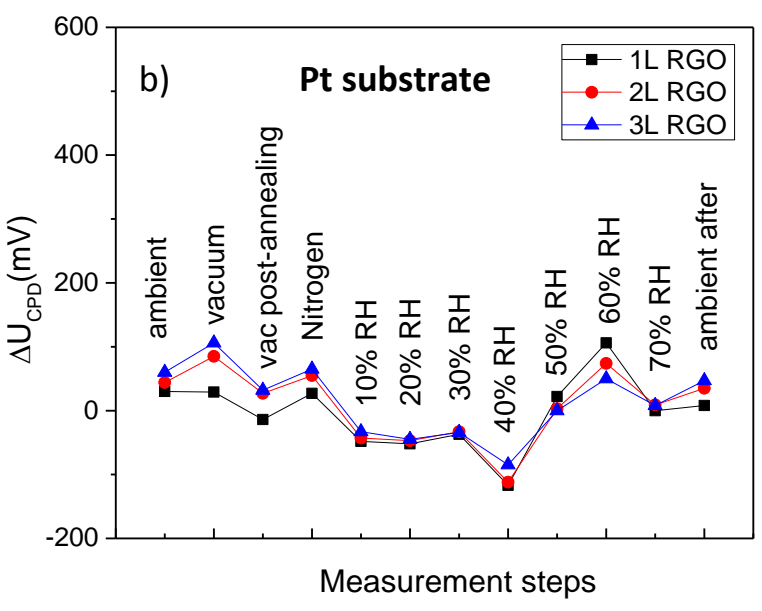

Figure 3: Summary of the relative change of surface potential values of RGO caused by the change in the humidity with respect to a) $\mathrm{Si}_{3} \mathrm{~N}_{4}$ and b) Pt substrates. Absolute values for the surface potential of RGO and the substrate, as well as the substrate are included in the S.I. section. 
We first analyse the case of RGO on $\mathrm{Si}_{3} \mathrm{~N}_{4}$, where the greatest change in the surface potential $(\sim 500 \mathrm{mV})$ occurs upon the transition between ambient and vacuum $\left(\mathrm{P} \approx 1 \times 10^{-5}\right.$ mbar) conditions, corresponding to desorption of loosely attached airborne adsorbates, in accordance with previously published results on various types of graphene [46, 47, 48, 49, 50]. This shows that RGO is sensitive to the water and other gases present in the ambient that alter the electronic properties of RGO. It should be noted that at this transition the contrast of the surface potential swaps for RGO on $\mathrm{Si}_{3} \mathrm{~N}_{4}$, with 1LRGO showing the lowest/highest values in ambient conditions and vacuum, respectively. This observation can be related to the fact that desorption of dopants occurs with different rate from domains of different thickness.

After thermal annealing in vacuum, at $150^{\circ} \mathrm{C}$, the contrast between domains of different thickness becomes more pronounced and the edges become more defined. At the same time, the surface potential drops slightly in comparison to the vacuum step, which could be associated with an increase in sample conductivity due to further reduction of functional groups. Introduction of water vapour into the chamber leads only to small variations of the surface potential values up to $40 \% \mathrm{RH}$. However, the $\Delta \mathrm{U}_{\mathrm{CPD}}$ values sharply decrease with humidity in the range 40-70 \% R.H., which could be attributed to the formation of a continuous layer of water and corresponding changes of work function.

For the majority of the environmental conditions studied here (i.e. from vacuum to $60 \%$ R.H.), the relative surface potential remains highest for 1LRGO, decreasing with the number of layers. It is noteworthy that, the surface potential remains homogeneous within each thickness domain (Figure $1 \mathrm{~b}-\mathrm{e}$ ), which is most likely due to the relatively good conductivity of RGO. The image obtained at $70 \%$ R.H. (Figure 1f), where the difference between the different domains becomes less clear might be attributed to a thin layer of water covering the whole sample and thus screening the underlying RGO at these conditions. This assumption is also confirmed by the fact that the contrast between individual domains is virtually lost at $70 \%$ R.H.

The $\Delta \mathrm{U}_{\mathrm{CPD}}$ reaches the value initially observed in ambient at $60-70 \%$ R.H., i.e. at humidity levels higher than typical ambient values of $40 \% \mathrm{RH}$. This fact indicates the importance of other dopants typically present in the ambient air that affect the electronic properties of graphene $[48,49,50]$.

Contrary to effects discussed above, in the case of RGO on Pt, $\Delta \mathrm{U}_{\mathrm{CPD}}$ values stay almost independent on the humidity conditions, oscillating between -100 and $+100 \mathrm{mV}$, with no clear effect of number of layers observed (Figure 2b).

\subsection{Graphene oxide - humidity variations}


The same experiment was conducted on the GO flakes. The image in Figure 4a shows the topography of the studied GO area. Different areas of the substrate, the border between them and the number of GO layers are indicated in the figure. Images $4 \mathrm{~b}-\mathrm{f}$ show surface potential maps of the GO area undergoing the same sequence of environmental conditions as shown previously for RGO.

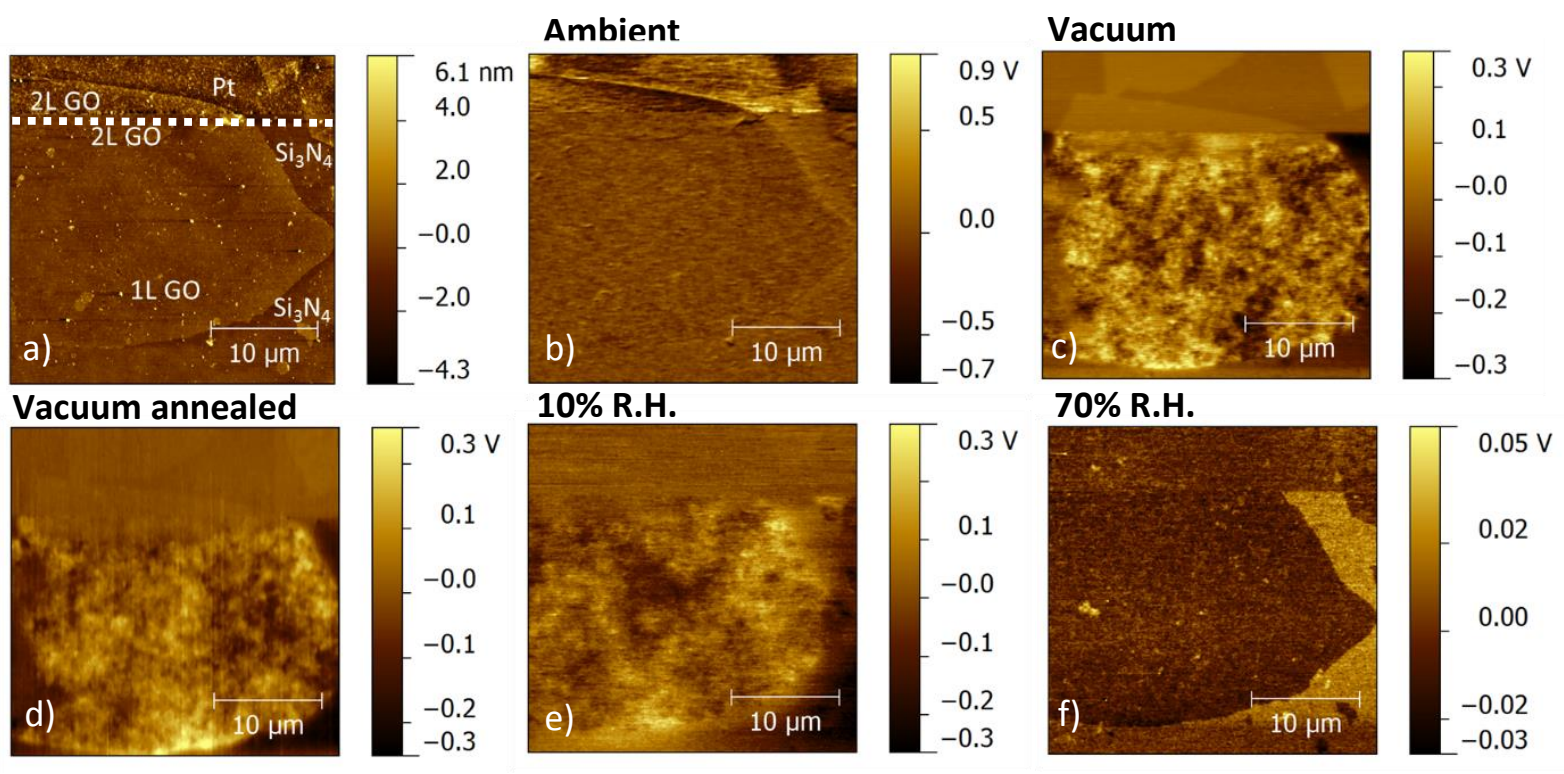

Figure 4: a) Topography and b-f) surface potential maps of GO on $\mathrm{Si}_{3} \mathrm{~N}_{4}$ and $\mathrm{Pt}$ at different humidity levels: b) ambient; c) vacuum; d) post-thermal annealing in vacuum; e) $10 \%$ R.H. and f) $70 \%$ R.H. Underlying substrate and number of GO layers are shown in a). Dashed line in a) indicates border between $\mathrm{Si}_{3} \mathrm{~N}_{4}$ and $\mathrm{Pt}$ areas of the supporting substrate. Note that the scale bars in b-f) are adapted to higlight individual features of the surface potential maps. All images are taken at room temperature. 
The contact potential difference data extracted from surface potential maps are summarised in Figure 5 for both $\mathrm{Si}_{3} \mathrm{~N}_{4}$ (Figure $5 \mathrm{a}$ ) and $\mathrm{Pt}$ (Figure $5 \mathrm{~b}$ ) areas of the supporting substrate.
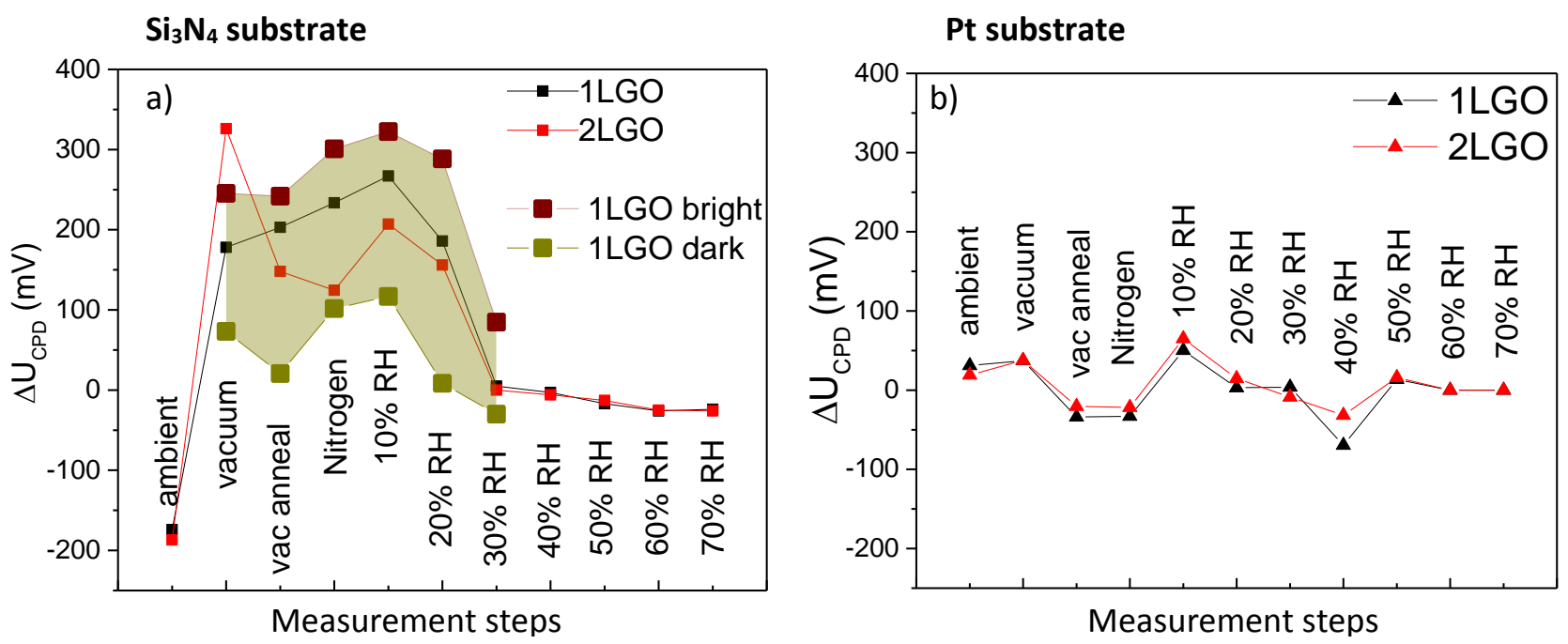

Figure 5: a) Summary of the relative change of the surface potential values of GO caused by the change in the humidity with respect to $\mathrm{Si}_{3} \mathrm{~N}_{4}$. The surface potential values are average values over the 1 and 2LGO layer areas displayed in the corresponding image. Grey filled area shows the range of surface potential non-uniformities with respect to $\mathrm{Si}_{3} \mathrm{~N}_{4}$. The associated values correspond to maximum brightness/darkness in each image. b) Summary of the relative change of the surface potential values of GO caused by the change in the humidity with respect to Pt. Absolute values for the surface potential of RGO and the substrate, as well as the substrate are included in the S.I. section.

In the case of GO flakes on $\mathrm{Si}_{3} \mathrm{~N}_{4}$ (Figure 5a), the biggest change in $\Delta \mathrm{U}_{\mathrm{CPD}}$ occurs upon transition from ambient to vacuum $\left(\mathrm{P} \approx 1 \times 10^{-5} \mathrm{mbar}\right)$ conditions, similar to the behaviour observed for RGO, showing also the inversion of contrast between substrates and GO layers. This indicates that GO's electronic properties are modified by water, gases and other volatile organic contaminants present in the ambient air. The change from ambient to vacuum is more pronounced for $2 \mathrm{LGO}$ than for $1 \mathrm{LGO}$. With the exception of the vacuum annealed and nitrogen steps, the overall trend observed with the change in humidity for $2 \mathrm{LGO}$ is similar with the one for $1 \mathrm{LGO}$.

Contrary to the RGO case (Figure 2c), removal of atmospheric contaminants in vacuum gives rise to non-uniformities in the surface potential of the $\mathrm{GO}$ on $\mathrm{Si}_{3} \mathrm{~N}_{4}$ scanned area and shows irregular domains of bright and dark contrast (Figure 4c), which are also observed at low humidity levels (Figure 4e). This is highlighted in Figure 5a, where the extreme values of 
the bright/dark patches are included for each measurement step from vacuum to $30 \%$ R.H. The bright and dark domains are attributed to charges trapped between the insulating GO and $\mathrm{Si}_{3} \mathrm{~N}_{4}$ substrate. This is consistent with the removal of p-type adsorbates in vacuum, as well as removal of other functional groups, partly reducing the GO and making it electrostatically transparent to substrate charges as previously observed on RGO samples in ambient [38]. Consistent with screening effects associated with thicker layers, patches in 2LGO appear less intense compared to $1 \mathrm{LGO}$ in vacuum, become very faint in the vacuum annealed case (as impurities in the substrate are desorbed) and are completely absent for $70 \% \mathrm{RH}$ due to screening effects associated with the water adsorbed on the GO sample.

The above claim is reaffirmed by the absence of this domain structure on GO/Pt area due to the metallic substrate acting as a drain electrode for charges. Above the $30 \%$ R.H. level, $\Delta \mathrm{U}_{\mathrm{CPD}}$ for $\mathrm{RGO}$ on Pt becomes again uniform showing almost constant values for all levels of humidity up to $70 \%$ R.H. Considering that GO is hydrophilic (i.e. more hydrophilic than RGO), it suggests that the GO flakes become completely covered by a water film at 30\% R.H., preventing additional changes at higher humidity levels. Indeed, as previously indicated, water forms hydrogen bonds with the hydroxyl and epoxy groups of GO, therefore absorbing significantly more water than pure graphene or RGO [51,52]. For the GO/Pt, $\Delta \mathrm{U}_{\mathrm{CPD}}$ values oscillate between $\pm 50 \mathrm{mV}$ (Figure $5 \mathrm{~b}$ ) with no clear dependence on the number of layers, similar to RGO case, where however a larger spread of data $\sim \pm 100 \mathrm{mV}$ was measured (Figure $3 b)$.

Summarising the two humidity experiments for GO and RGO supported on $\mathrm{Si}_{3} \mathrm{~N}_{4}$, the main difference is that the surface potential reaches the values observed in ambient at different humidity levels: $\sim 60 \%$ R.H for RGO and $\sim 30 \%$ R.H. for GO, in line with the mainstream understanding that GO is more hydrophilic than RGO and formation of a continuous water layer occurs at lower humidity level on GO as compared to RGO due to the presence of functional groups. When deposited on Pt, apart from slightly larger surface potential variations for RGO, no significant differences are noted for both $\mathrm{GO}$ and RGO with the change in humidity, indicating that the electronic properties of GO and RGO on Pt are not affected by water vapours.

Our results clearly indicate that the substrate plays an important role and that complex mechanisms are governing the interaction between water molecules and GO and RGO. The role of the substrate was highlighted previously for pristine graphene on $\mathrm{Si} / \mathrm{SiO}_{2}$ [53], where it was shown that the effect of water strongly depends on the properties of the substrate. Here, the dipole moments of water adsorbates cause local electrostatic fields that can shift the 
substrate's defect states with respect to the Fermi energy of graphene and cause doping. Moreover, according to previous studies, it was postulated that the contact angle of water on graphene is dependent on both the liquid - graphene and liquid - substrate interactions, resulting in different degrees of wetting transparency of graphene [54]. As such, graphene is more transparent to wetting on hydrophilic substrates, but more opaque to wetting on hydrophobic substrates. It was also shown that few layer graphene does not significantly change the wettability of gold, silicon and copper, and few layer graphene appears hydrophilic in contradiction to bulk graphite [55]. The contact angles for $\mathrm{Si}$ and $\mathrm{Au}$ were measured $\sim 32.6^{\circ}$ and $77.4^{\circ}$, respectively, with a monolayer graphene coating causing only a $\sim 1-2 \%$ increase in the contact angles when deposited on $\mathrm{Si}$ and $\mathrm{Au}$ [55]. $\mathrm{Si}_{3} \mathrm{~N}_{4}$ versus $\mathrm{Pt}$ shows similar behaviour to $\mathrm{Si}$ versus $\mathrm{Au}$, as the contact angles indicate hydrophilic character for $\mathrm{Si}_{3} \mathrm{~N}_{4}$, with contact angle $\sim 33^{\circ}$ (similar to $\mathrm{Si}$ ) [56] and 50 for Pt [57]. Following the same argument, it can thus be assumed that graphene, and also RGO, which has a reasonable structural and electrical resemblance to graphene, will show a more hydrophilic character on the $\mathrm{Si}_{3} \mathrm{~N}_{4}$ substrate, which is more hydrophilic than Pt. This is in line with our observations of more pronounced changes in humidity for $\mathrm{RGO}$ placed on $\mathrm{Si}_{3} \mathrm{~N}_{4}$ compared to Pt substrates. Although a similar trend is observed for $\mathrm{GO}$, the analysis is more complicated as the functional groups make the surface strongly hydrophilic hindering the effect of the substrate.

Irrespective of the substrate, both GO and RGO show a similar trend for 1, 2 and 3 layers when subject to humidity variations, indicating that, for example for GO-based humidity sensors thickness is not an important factor, at least for the thickness range investigated in this paper. This is in contrast to studies of humidity effects on epitaxial graphene on SiC and CVDgrown graphene, which pointed to increased sensitivity with decreasing number of layers [48, $49,50]$, with single-layer graphene being the most sensitive to environment changes.

\section{3 "Soft" reduction of GO during vacuum annealing}

As shown above, uniform surface potential maps are obtained for both GO and RGO on Pt, whereas non-uniform distribution $\Delta \mathrm{U}_{\mathrm{CPD}}$ is observed for $\mathrm{GO}$ on $\mathrm{Si}_{3} \mathrm{~N}_{4}$, due to trapped charges. This is not, however, the case for $\mathrm{RGO} / \mathrm{Si}_{3} \mathrm{~N}_{4}$, owing to a more conductive nature of RGO. To further investigate the nature of the observed patches, we study the effect of different vacuum annealing temperatures on the surface potential of GO. Figure 6a shows topography image of a GO area with marked regions of different number of layers. After obtaining topography and initial surface potential maps at room temperature (Figure 6b), the sample was sequentially annealed in vacuum for 1 hour at each temperature: $50^{\circ} \mathrm{C}, 100^{\circ} \mathrm{C}$ and $150^{\circ} \mathrm{C}$, then allowed to 
cool down to room temperature to record the surface potential maps. Surface potential maps of GO for annealing temperatures of up to $100^{\circ} \mathrm{C}$ (Figure $6 \mathrm{c}-\mathrm{d}$ ) show almost no difference with respect to room temperature (Figure 6b), however a decrease in surface potential values is observed for 1 and 2LGO with increase of temperature. The surface potential of 1LGO changes at $150^{\circ} \mathrm{C}$, where the patches disappear and the surface potential becomes effectively uniform as observed for RGO, likely to be consistent with GO chemical reduction when approaching $150^{\circ} \mathrm{C}$. However, this effect is only temporary as re-exposing the sample to ambient after the vacuum thermal treatment at $150^{\circ} \mathrm{C}$ brings back the non-uniformities in surface potential (see details in S.I. section), due to either restored environmental doping trapped between the GO flake and the substrate or accumulated charges in $\mathrm{Si}_{3} \mathrm{~N}_{4}$. The variation of GO surface potential as a result of annealing is furthermore illustrated by representative cross-sectional profiles taken at room temperature, $50^{\circ} \mathrm{C}, 100^{\circ} \mathrm{C}$ and $150^{\circ} \mathrm{C}$ for $\mathrm{GO}$ on $\mathrm{Si}_{3} \mathrm{~N}_{4}$ (Figure $6 \mathrm{~g}$ ) and $\mathrm{Pt}$ substrates (Figure 6h).
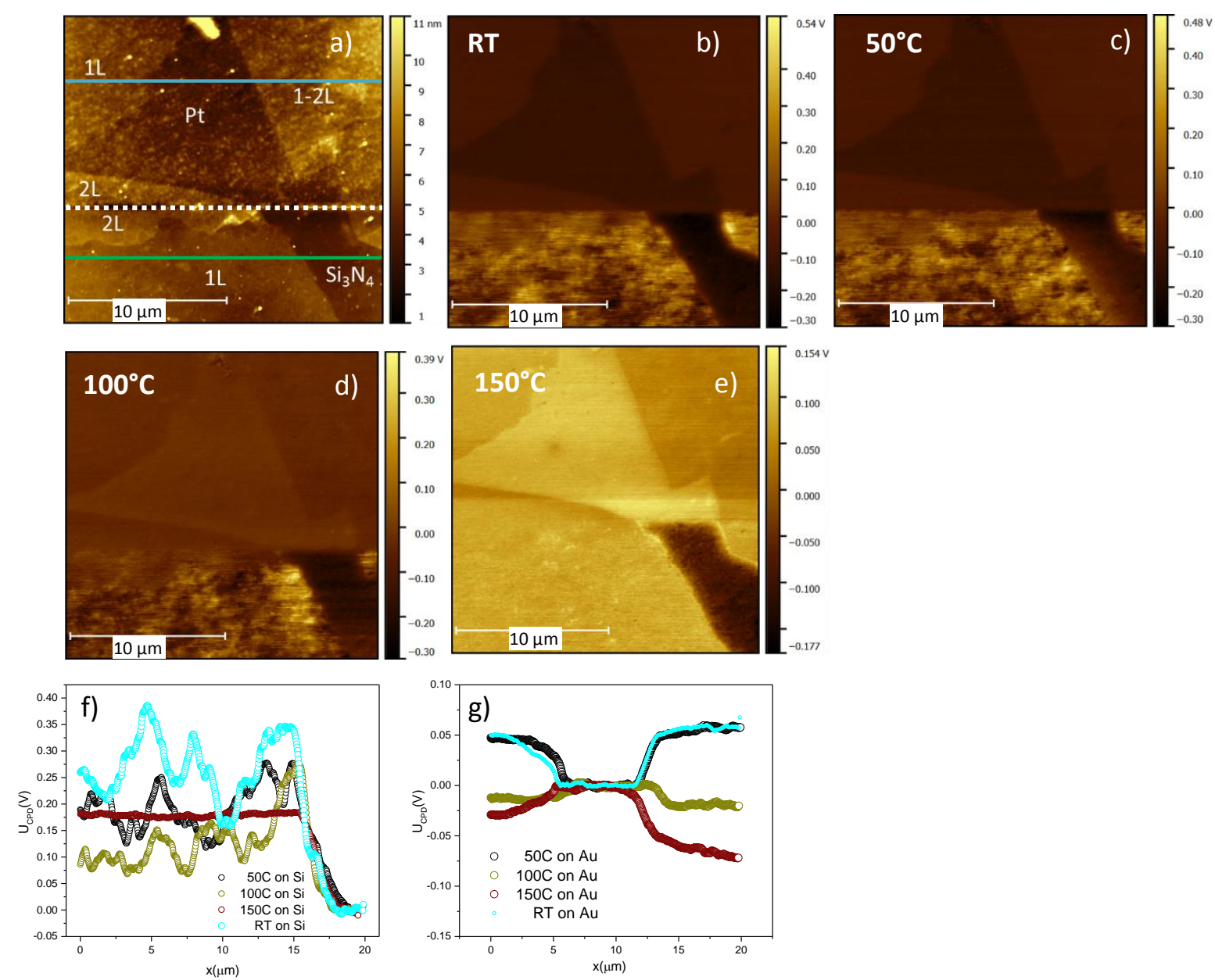

Figure 6: a) Topography; b-e) surface potential maps of GO taken in vacuum: b) initial state prior to annealing and c-e) after annealing at c) $50{ }^{\circ} \mathrm{C}$, d) $100{ }^{\circ} \mathrm{C}$, e) $150{ }^{\circ} \mathrm{C}$. All measurements 
were taken at room temperature. Dashed line in a) indicates a border between $\mathrm{Si}_{3} \mathrm{~N}_{4}$ and $\mathrm{Pt}$ substrates. f), g) profiles of surface potential as measured at different temperatures. Profiles are taken along the solid blue lines (panel a): g) $\mathrm{GO} / \mathrm{Si}_{3} \mathrm{~N}_{4}$ and h) GO/Pt.

\section{Conclusions}

The electronic properties of GO and RGO sheets deposited on metallic (Pt) and semiconducting $\left(\mathrm{Si}_{3} \mathrm{~N}_{4}\right)$ substrates have been investigated in controlled humidity environment. It is found that the supporting substrate plays an important role, with Pt making both GO and RGO insensitive to humidity variations and change in environment, from ambient to vacuum. Significant variations in surface potential with the humidity and environment are observed when GO and RGO sheets are placed on $\mathrm{Si}_{3} \mathrm{~N}_{4}$, with the greatest change occurring for the transition between ambient and vacuum environment. The surface potential reaches the values observed initially in ambient at: $\sim 60 \%$ R.H for RGO and $\sim 30 \%$ for GO, in agreement with the conventional understanding that GO is more hydrophilic than RGO and that water saturation for GO occurs in lower humidity environment compared to that of RGO due to the presence of functional groups. Unlike epitaxial and CVD-grown graphene where the response to water and change in environment is strongly thickness dependent, GO and RGO do not exhibit significant thickness dependency for 1, 2 or 3 layers, indicating that the GO/RGO film thickness should not be a concern for humidity sensor applications. At the same time, we note that the electronic behaviour of GO and RGO is also affected by contaminants commonly present in ambient air, such as volatile organic compounds or gases and these effects should be taken into account for sensors designed to work in the ambient. The study provides valuable information for GO/RGO sensor design to ensure optimum sensitivity and reliability and will aid in developing models for realistic sensors.

Acknowledgments NPL authors acknowledge financial support from the UK's National Measurements Service under SC Graphene Project and the Graphene Flagship (no. CNECTICT-604391).

\section{Supplementary information}

1. SKPM on GO in ambient after thermal treatment in vacuum

SKPM in ambient was carried out on GO after thermal annealing in vacuum at $150{ }^{\circ} \mathrm{C}$. The surface potential map and the topography is shown in Figure S1, where patches are observed 
in the surface potential map on the 1LGO region, indicating restoration of GO properties and only temporary effect of reduction.
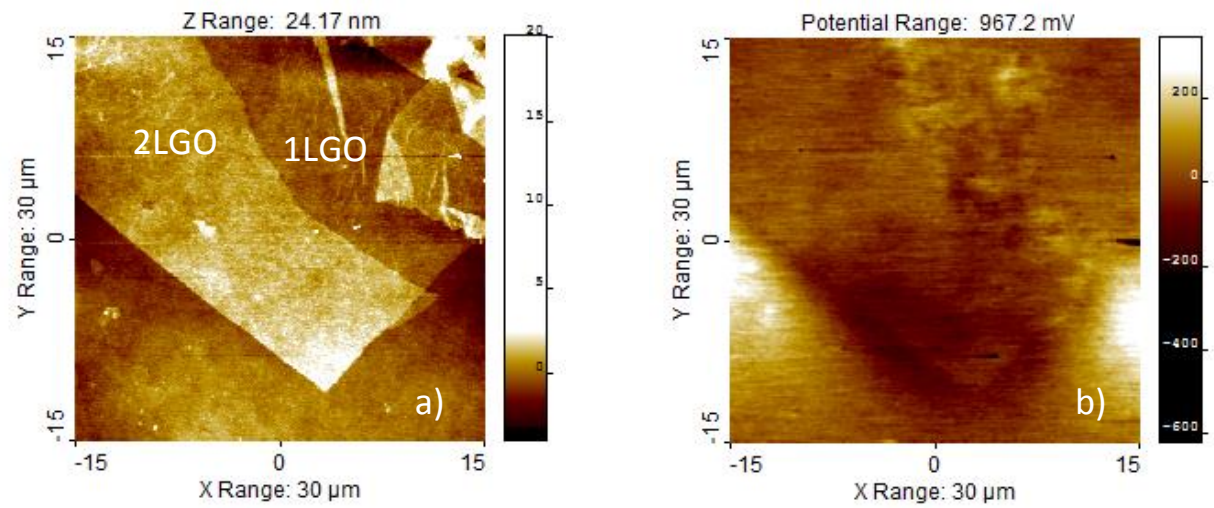

Figure S1: a) Topography and b) surface potential of GO in ambient after annealing of the sample at $150{ }^{\circ} \mathrm{C}$.

2. Raman spectroscopy of GO and RGO (reduced at $300^{\circ} \mathrm{C}$ )

Raman intensity maps for the 2D-, G- and D-band were acquired on GO and RGO flakes supported on $\mathrm{Si}_{3} \mathrm{~N}_{4}$ and are presented in Figure $\mathrm{S} 2 \mathrm{a}$ and $\mathrm{b}$. 

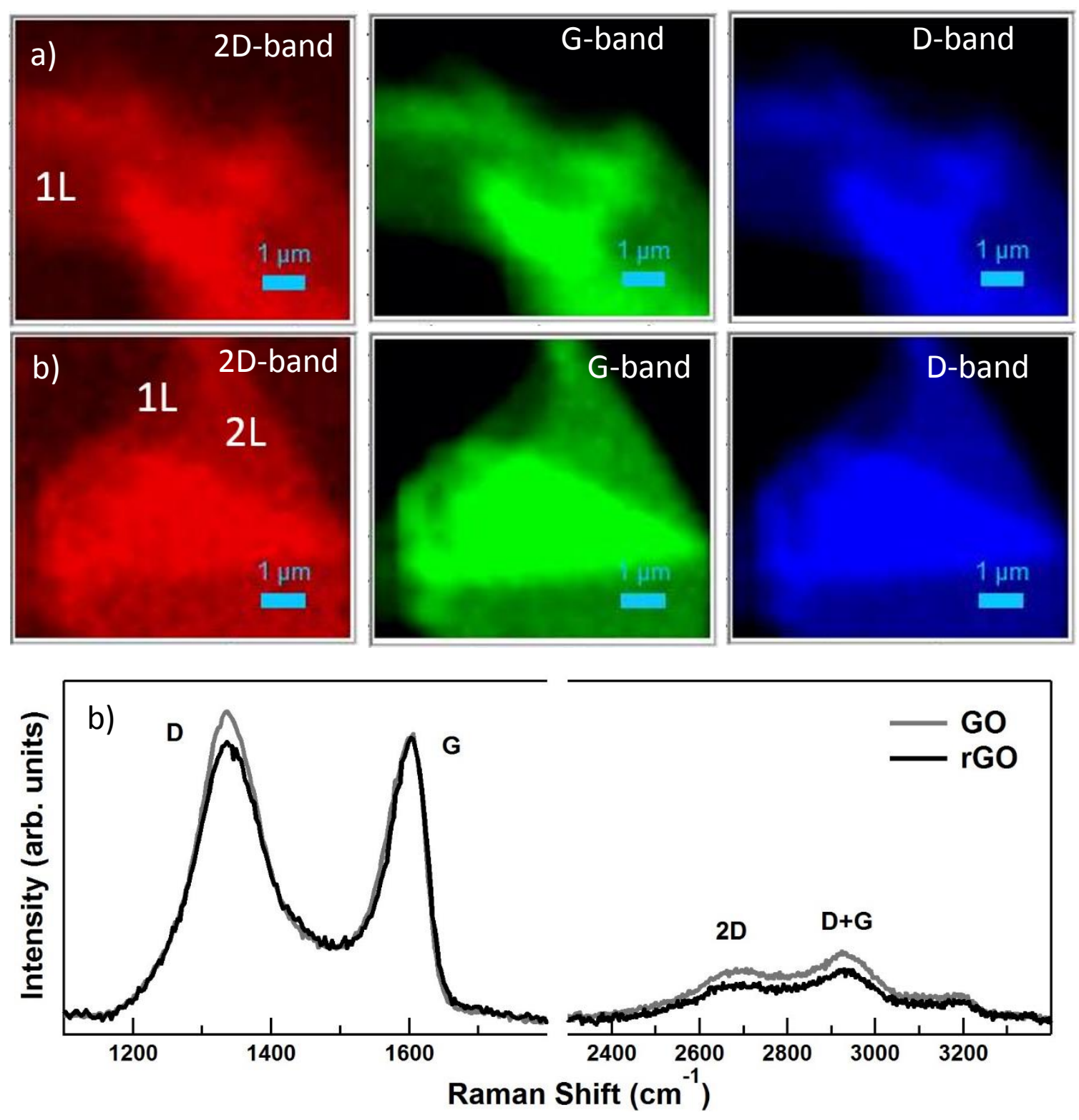

Figure S2: Raman intensity maps for a) $\mathrm{GO}$ and b) RGO flakes on $\mathrm{Si}_{3} \mathrm{~N}_{4}$ substrate. The red intensity map is associated with the $2 \mathrm{D}$ peak, the green with the $\mathrm{G}$ peak and the blue with the D peak. (c) Representative Raman spectra for GO and RGO samples, showing D- and G-band region, and the $2 \mathrm{D}$ region.

All intensity maps display a uniform response for GO and RGO monolayer regions and show a clear increase in intensity with the layer thickness.

Individual Raman spectra extracted from preselected regions of monolayer flakes display two prominent peaks corresponding to the D- and G-band, respectively, as illustrated in Figure S2c. The position and full-width-at-half-maximum (FWHM) of the D- and G-peak does not depend on the number of layers. For GO, the D and G peaks appear at $\sim 1341 \mathrm{~cm}^{-1}$ and $\sim 1593 \mathrm{~cm}^{-1}$, 
and for RGO they appear at $\sim 1342 \mathrm{~cm}^{-1}$ and at $\sim 1597 \mathrm{~cm}^{-1}$, respectively. The D-peak position remains relatively constant upon annealing, whereas the G-peak shifts to higher frequency for RGO, which is consistent with charge transfer between the flake and the substrate. The increase in crystalline order upon annealing is supported by the decrease of the FWHM of the G, from $75 \mathrm{~cm}^{-1}$ in the case of GO to $67 \mathrm{~cm}^{-1}$ for RGO. The intensity ratio of D- with respect to G-band is a measure of disorder and a slight decrease of $\mathrm{I}_{\mathrm{D}} / \mathrm{I}_{\mathrm{G}}$ is observed upon thermal reduction, from 1.1 to 0.9 .

The 2D-band region for both GO and RGO shows three broad and low intensity peaks, which is significantly different from that of exfoliated monolayer graphene, dominated by a single and narrow 2D peak. Apart from the 2D peak, at $\sim 2702 \mathrm{~cm}^{-1}$ for $\mathrm{GO}$, two additional peaks at $\sim 2936 \mathrm{~cm}^{-1}$ and at $\sim 3202 \mathrm{~cm}^{-1}$ are apparent, previously associated with disorder [38, 46, 47]. Upon reduction, peaks in the $2 \mathrm{D}$ region shift to lower frequency: $\sim 2672 \mathrm{~cm}^{-1}$ for the $2 \mathrm{D}$ peak and $2931 \mathrm{~cm}^{-1}$ and $3189 \mathrm{~cm}^{-1}$, respectively for the two additional contributions.

$\mathrm{U}_{\mathrm{CPD}}$ values for RGO on $\mathrm{Pt}$ and $\mathrm{Si}_{3} \mathrm{~N}_{4}$ are summarised in Figure $\mathrm{S} 3$ and for GO in Figure S4.
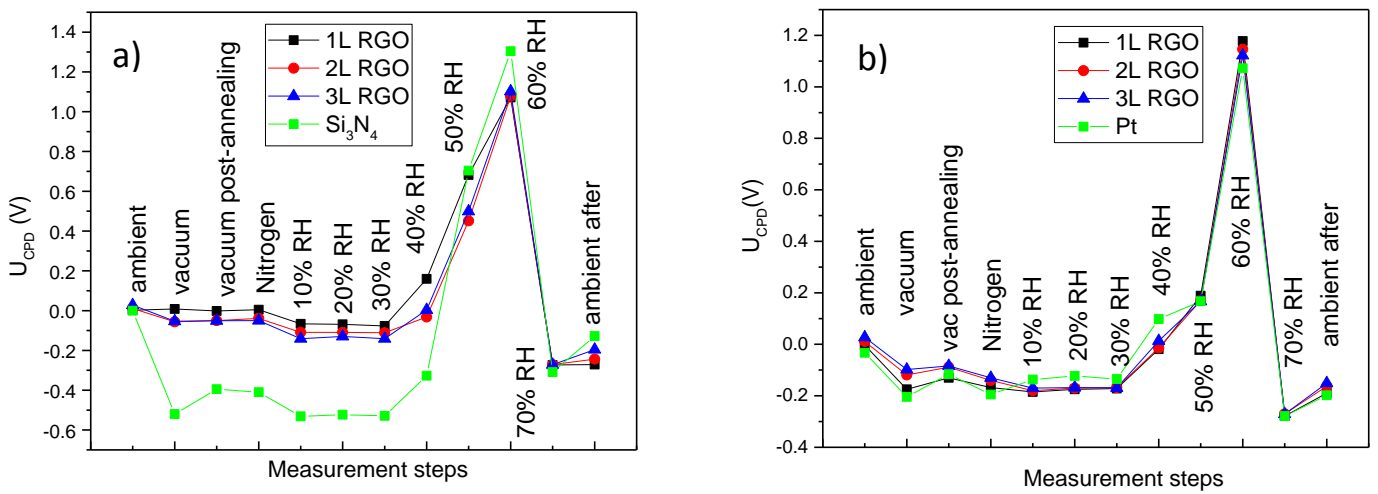

Figure S3 Absolute values for the measurement sequence of RGO on (a) $\mathrm{Si}_{3} \mathrm{~N}_{4}$ and (b) Pt substrates.
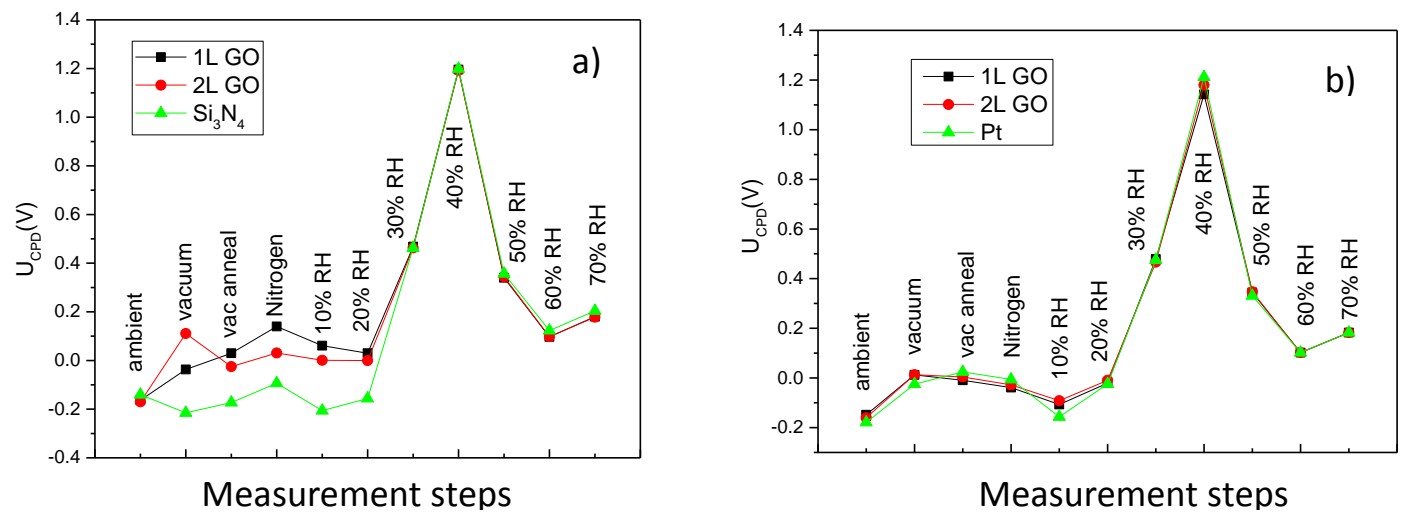

Figure S4 Absolute values for the measurement sequence of $\mathrm{GO}$ on (a) $\mathrm{Si}_{3} \mathrm{~N}_{4}$ and (b) $\mathrm{Pt}$ substrates. 


\section{References}

1. Kian Ping L, Qiaoliang B, Eda G, Chhowalla M. Graphene oxide as a chemically tunable platform for optical applications. Nat. Chem., 2010; 2, 1015-24

2. Park S, Ruoff RS. Chemical methods for the production of graphenes. Nat. Nanotechnol., $2009 ; 4,217-24$

3. He HY, Klinowski J, Forster M, Lerf A. A new structural model for graphite oxide. Chem. Phys. Lett., 1998; 287, 53-6

4. Gao W, Alemany LB, Ci L, Ajayan PM. New insights into the structure and reduction of graphite oxide. Nat. Chem. 2009; 1, 403-8

5. Perrozzi, F., Prezioso, S., Ottaviano, L. Graphene oxide : from fundamentals. Journal of Physics: Condensed Matter., 2015; 27, 13002. doi:10.1088/0953-8984/27/1/013002

6. Nair, R. R.; Wu, H. A.; Jayaram, P. N.; Grigorieva, I. V.; Geim, A. K. Unimpeded Permeation of Water through Helium- Leak-Tight Graphene-Based Membranes. Science, $2012 ; 335,442-444$

7. Chandra, V., Park,J., Chun, Y., Lee, J. W., Hwang, I.-C., Kim, K.S., Water-dispersible magnetite-reduced graphene oxide composites for arsenic removal. ACS Nano; 2010, 4, 3979

8. Zhu, J., Sadu, R., Wei, S., Chen, D.H., Haldolaarachchige, N., Luo, Z., et al., Magnetic Graphene Nanoplatelet Composites toward Arsenic Removal, ECS J. Solid State Sci. Technol., 2012; 1, M1-M5

9. Kemp, K.C., Seema, H., Saleh, M., Le, N.H., Mahesh, K., Chandra, V., et al., Environmental applications using graphene composites: water remediation and gas adsorption. Nanoscale, $2013 ; 5,3149$

10. Robinson JT, Keith Perkins F, Snow E S, Wei Z and Sheehan P E, Reduced graphene oxide molecular sensors, Nano Lett., 2008; 8 3137-40

11. Fowler J D, Allen MJ, Tung V C, Yang Y, Kaner R B and Weiller B H., Practical chemical sensors from chemically derived graphene ACS Nano, 2009; 3 301-6

12. Jung I, Dikin D, Park S, Cai W, Mielke S L and Ruoff R S Effect of water vapor on electrical properties of individual reduced graphene oxide sheets J. Phys. Chem. C, 2008; 112 20264-8

13. Prezioso S, Perrozzi F, Giancaterini L, Cantalini C, Treossi E, Palermo V, Nardone M, Santucci S and Ottaviano L Graphene oxide as a practical solution to high sensitivity gas sensing J. Phys. Chem. C, 2013; 117 10683-90 
14. L. Huang, ZH. Wang, J. Zhang, J. Pu, Y. Lin, S. Xu, et al., Fully Printed, Rapid-Response Sensors Based on Chemically Modified Graphene for Detecting NO2 at Room Temperature. ACS Appl. Mater. Interfaces, 2014; 6, 7426-7433

15. Hu, N., Yang, Z., Wang, Y., Zhang, L. \& Wang, Y. Ultrafast and sensitive room temperature NH 3 gas sensors based on chemically reduced graphene oxide. Nanotechnology, $2014 ; 25,025502$

16. Yun, Y. J, W. G. Hong, N.-J. Choi, B. H. Kim, Y. Jun, and \& H.-K. Lee, Ultrasensitive and highly selective graphene-based single yarn for use in wearable gas sensor. Sci. Rep., 2015; 5, 10904

17. Cherenack, K., van Pieterson, L. Smart textiles: Challenges and opportunities. J. App. Phys. 2012; 112, 091301

18. Guo, Y., Wu, B., Liu, H., Ma, Y., Yang, Y., Zheng, J. et al. Electrical Assembly and Reduction of Graphene Oxide in a Single Solution Step for Use in Flexible Sensors. Adv. Mater. , 2011; 23, 4626-4630

19. Ohno, Y.; Maehashi, K.; Yamashiro, Y.; Matsumoto, K. Electrolyte-Gated Graphene FieldEffect Transistors for Detecting pH and Protein Adsorption. Nano Lett., 2009; 9, 3318-3322

20. Min, S. K.; Kim, W. Y.; Cho, Y.; Kim, K. S. Fast DNA Sequencing with a Graphene-Based Nanochannel Device. Nat. Nanotechnol., 2011; 6, 162

21. Tiwari, J.N., Vij, V., Kemp, K. C., Kim, K. S. Engineered Carbon-Nanomaterial-Based Electrochemical Sensors for Biomolecules, ACS Nano, 2016; 10 (1), 46-80

22. Rauti, R., Lozano Valdes, N.V., Leon, V., Scaini, D., Musto, M., Rago, I. et al., Graphene Oxide Nanosheets Reshape Synaptic Function in Cultured Brain Networks ASC Nano, 2016; DOI: 10.1021/acsnano.6b00130

23. Guo, C. X.; Ng, S. R.; Khoo, S. Y.; Zheng, X.; Chen, P.; Li, C. M. RGD-Peptide Functionalized Graphene Biomimetic Live-Cell Sensor for Real-Time Detection of Nitric Oxide Molecules. ACS Nano, 2012; 6 (8), pp 6944-6951.

24. Nguyen P., Berry V., Graphene interfaced with biological cells: opportunities and challenges J. Phys.Chem. Lett. 2012; 3 1024-9

25. Wang $\mathrm{H}$, Wu Y, Cong C, Shang J and Yu T, Hysteresis of electronic transport in graphene transistors ACS Nano, 2010; 4 7221-8

26. Yao, Y.; Chen, X.; Zhu, J.; Zeng, B.; Wu, Z.; Li, X. The Effect of Ambient Humidity on the Electrical Properties of Graphene Oxide Films. Nanoscale Res. Lett. 2012; 7, 363

27. Yao, Y.; Chen, X.; Guo, H.; Wu, Z. Graphene Oxide Thin Film Coated Quartz Crystal Microbalance for Humidity Detection. Appl. Surf. Sci. 2011; 257, 7778-7782 
28. Yao, Y.; Chen, X.; Guo, H.; Wu, Z.; Li, X. Humidity Sensing Behaviors of Graphene Oxide-Silicon Bi-layer Flexible Structure. Sens. Actuators, B 2012; 161, 1053-1058

29. Borini, S., White, R., Wei, D., Astley, M., Haque, S., Spigone, E., Harris, N. (2013). Ultrafast Graphene Oxide Humidity Sensors ACS Nano 2013; 7, 11166-11173

30. Burnett, T., Yakimova, R., Kazakova, O. (2011). Mapping of Local Electrical Properties in Epitaxial Graphene Using Electrostatic Force Microscopy. NanoLetters, 2011; , 11, 2324

31. Kazakova, O., Burnett, T. L., Patten, J., Yang, L., Yakimova, R. Epitaxial graphene on SiC (000-1): functional electrical microscopy studies and effect of atmosphere, Nanotechnology 2013; 24, 215702. doi:10.1088/0957-4484/24/21/215702

32. Panchal, V., Pearce, R., Yakimova, R., Tzalenchuk, A., Kazakova, O., Standardization of surface potential measurements of graphene domains," Scientific Reports, 2013; 3, 2597-8.

33. Kazakova, O., Panchal, V., Burnett, T. L. Epitaxial Graphene and Graphene-Based Devices Studied by Electrical Scanning Probe Microscopy, Crystals, 2013; 3, 191-233

34. Robinson, B. J., Giusca, C. E., Gonzalez, Y. T., Kay, N. D., Kazakova, O. Structural, optical and electrostatic properties of single and few- layers MoS2: effect of substrate. 2D Materials, 2015; 2(1), 15005. doi:10.1088/2053-1583/2/1/015005

35. Melios, C., Panchal, V., Giusca, C. E., Strupiński, W., Silva, S. R. P., Kazakova, O. Carrier type inversion in quasi-free standing graphene: studies of local electronic and structural properties. Sci Reports, 2015; 5, 10505 DOI: 10.1038/srep10505

36. Liscio, A., Veronese, G.P., Treossi, E., Suriano, F., Rossella, F., Bellani, V., et al., Charge transport in graphene - polythiophene blends as studied by Kelvin Probe Force Microscopy and transistor characterization, J. Mater. Chem., 2011; 21, 2924

37. Jaafar, M., López-Polín, G., Gómez-Navarro, C., Gómez-Herrero, J., Step like surface potential on few layered graphene oxide Step like surface potential on few layered graphene oxide, Appl. Phys. Lett., 2012; 101, 263109; doi: 10.1063/1.4773357

38. Giusca, C. E., Perrozzi, F., Melios, C., Treossi, E., Palermo, V., Kazakova, O., \& Ottaviano, L.. Electrostatic transparency of graphene oxide sheets. CARBON, 2015; 86, 188-196. doi:10.1016/j.carbon.2015.01.035

39. Yan, L., Punckt, C., Aksay, I. A., Mertin, W., \& Bacher, G. (2011). Local Voltage Drop in a Single Functionalized Graphene Sheet Characterized by Kelvin Probe Force Microscopy, NanoLett, 2011; 11, 3543-3549.

40. Mishra, M., Joshi, R. K., Ojha, S., Kanjilal, D., \& Mohanty, T. (2013). Role of Oxygen in the Work Function Modi fi cation at Various Stages of Chemically Synthesized Graphene, J. Phys. Chem. C, 2013; 117, 19746-19750 
41. Li, J., Qi, X., Hao, G., Ren, L., Zhong, J., In-situ investigation of graphene oxide under UV irradiation: Evolution of work function In-situ investigation of graphene oxide under UV irradiation: Evolution of work function, AIP Advances, 2015; 5, 067154

42. Saloma, F. C., Lanzoni, E. M., Costa, C. A., Deneke, C. \& Barros, E. B. Determination of High-Frequency Dielectric Constant and Surface Potential of Graphene Oxide and Influence of Humidity by Kelvin Probe Force Microscopy. Langmuir, 2015; 1-5, doi:10.1021/acs.langmuir.5b01786

43. Kehayias, C. E., Macnaughton, S., Sonkusale, S. \& Staii, C. Kelvin probe microscopy and electronic transport measurements in reduced graphene oxide chemical sensors. Nanotechnology, 2013; 24, 245502

44. Lee, C. W., Min, B. J., In, S. \& Jeong, H. K. Stacking of water molecules in hydrophilic graphene oxides characterized by Kelvin probe force microscopy. Carbon 2012; 54, 353-358 45. Hirata, M.; Gotou, T.; Horiuchi, S.; Fujiwara, M.; Ohba M., Thin-film particles of graphite oxide 1:: High-yield synthesis and flexibility of the particles, Carbon 2004; 42, 2929-37

46. Martins Ferreira, E.H.; Moutinho, M. V. O.; Stavale, F.; Lucchese, M. M.; Capaz, R. B.; Achete, C. A.; Jorio, A., Evolution of the Raman spectra from single-, few-, and many-layer graphene with increasing disorder, Physical Review B 2010; 82, 125429

47. Cancado, L. G.; Jorio, A.; Martins Ferreira, E. H.; Stavale, F.; Achete, C. A.; Capaz, R. B.; Moutinho, M. V. O.; Lombardo, A.; Kulmala, T. S.; Ferrari, A. C., Quantifying Defects in Graphene via Raman Spectroscopy at Different Excitation Energies, Nano Letters 2011; 11, 3190-3196

48. Giusca, C.E., Panchal, V., Munz, M., Wheeler, V.D. Nyakiti, L.O., Myers-Ward, R.L., et al., Water Affinity to Epitaxial Graphene: The Impact of Layer Thickness, Adv. Mat. Interfaces, 2015; 5, 1500252

49. Panchal, V; Giusca, CE; Lartsev, A; Martin, NA; Cassidy, N; Myers-Ward, RL; Gaskill, DK; Kazakova, O., Atmospheric doping effects in epitaxial graphene: correlation of local and global electrical studies 2D MATERIALS 2016; 3, 1, 015006

50. Melios, C; Centeno, A; Zurutuza, A; Panchal, V; Giusca, CE; Spencer, S; Silva, SRP; Kazakova, O Effects of humidity on the electronic properties of graphene prepared by chemical vapour deposition CARBON 2016; 103 273-80

51. Toda, K., Furue, R., Hayami, S., Recent Progress in Applications of Graphene Oxide for Gas Sensing: A Review. Analytica Chimica Acta, 2015; 878, 43-53 
52. Guo, L., Jiang, H.-B., Shao, R.-Q.,Zhang, Y.-L., Xie, S.-Y., Wang, J.-N., et al., Two-beamlaser interference mediated reduction, patterning and nanostructuring of graphene oxide for the production of a flexible humidity sensing device. Carbon 2012; 50, 1667-1673

53. Wehling, T. O., Lichtenstein, A. I., Katsnelson, M. I., First-principles studies of water adsorption on graphene: The role of the substrate. Applied Physics Letters, 2008; 93(20), 202110. doi:10.1063/1.3033202

54. Shih, C.-J., Strano, M. S., Blankschtein, D. Wetting translucency of graphene. Nature Materials, 2013; 12(10), 866-9. doi:10.1038/nmat3760

55. Rafiee, J., Mi, X., Gullapalli, H., Thomas, A. V, Yavari, F., Shi, Y., et al., Wetting transparency of graphene. Nature Materials, 2012; 11(3), 217-22. doi:10.1038/nmat3228

56. Diao, J., Ren, D., Engstrom, J.R., Lee, K.H., A surface modification strategy on silicon nitride for developing biosensors, Anal. Biochem. 2005; 343(2), 322-8

57. Bernardin, J.D., Mudawar, I., Walsh, C.B., Franses, E.I., Contact angle temperature dependence for water droplets on practical aluminum surfaces, Int. J. Heat Mass Trasfer, 1997; $40,5,1017-33$ 